\title{
Observations of oxidation mechanisms and kinetics in faceted FeCo magnetic nanoparticles
}

\author{
N. J. Jones, ${ }^{1, a)}$ K. L. McNerny, ${ }^{1}$ A. T. Wise, ${ }^{2,3}$ M. Sorescu, ${ }^{4}$ M. E. McHenry, ${ }^{1,3}$ and \\ D. E. Laughlin ${ }^{1,2,3}$ \\ ${ }_{1}^{1}$ Department of Materials Science and Engineering, Carnegie Mellon University, Pittsburgh, Pennsylvania \\ 15213, USA \\ ${ }^{2}$ Department of Electrical and Computer Engineering, Carnegie Mellon University, Pittsburgh, \\ Pennsylvania 15213, USA \\ ${ }^{3}$ Data Storage Systems Center, Carnegie Mellon University, Pittsburgh, Pennsylvania 15213, USA \\ ${ }^{4}$ Department of Physics, Duquesne University, Pittsburgh, Pennsylvania 15282, USA
}

(Presented 19 January 2010; received 3 November 2009; accepted 18 November 2009; published online 15 April 2010)

$\left(\mathrm{Fe}_{50} \mathrm{Co}_{50}\right)_{97} \mathrm{~V}_{2} \mathrm{Nb}_{1}$ nanoparticles were synthesized in an induction plasma torch and oxidized isochronally at temperatures between room temperature (RT) and $900{ }^{\circ} \mathrm{C}$. The particles exhibited three stages of oxidation present at different temperatures. The initial oxide layer was nearly Co-free, beginning at $3 \mathrm{~nm}$ at RT, and Co appeared to oxidize separately from the iron. Iron cations were determined to be the mobile species during oxidation, yielding a progressively more Co-rich core as oxidation progressed, decreasing from an average diameter of 42 to $25 \mathrm{~nm}$, while the oxide tripled in size. At $350{ }^{\circ} \mathrm{C}$, the particles exhibited a less dense core, and at $900{ }^{\circ} \mathrm{C}$ the particles were observed to have changed morphology completely with some sintering, forming completely oxidized particles with an average diameter three times the as received size. (C) 2010 American Institute of Physics. [doi:10.1063/1.3334197]

\section{INTRODUCTION}

FeCo nanoparticles are important materials in biomedical, electromagnetic interference (EMI) absorption, and other applications. ${ }^{1,2}$ To understand their performance in varying environmental conditions, their oxidation behavior needs to be investigated, noting how the oxide couples to the core of FeCo.

FeCo magnetic nanoparticles have the highest room temperature (RT) saturation magnetization, lying at the peak of the Slater-Pauling curve. ${ }^{3,4}$ When used in applications, therefore, one can often get the same property performance as other materials, while using a lower concentration of the nanoparticles. A high saturation induction along with a high anisotropy field is essential to high frequency applications of nanoparticles for use in gigahertz frequency EMI absorption. ${ }^{5}$ The anisotropy field can be increased by increasing the magnetocrystalline anisotropy of a material, which can be accomplished in $\mathrm{FeCo}$ nanoparticles by oxidation. For example, if $\mathrm{Co}$ is incorporated in the oxide, large magnetic anisotropies associated with Co-ferrite can be realized. If the oxide is well lattice-matched to the core, exchange bias effects can be observed. ${ }^{6}$ This oxidation, both compositionally and kinetically, depends on the surface orientation of the faceted nanoparticles. While bulk studies have been done on this alloy system, more in depth nanoparticle analyses have yet to be completed. ${ }^{7}$ A general study of the oxidation kinetics and morphology of these types of nanoparticles is reported below, from RT to $900{ }^{\circ} \mathrm{C}$, using multiple experimental techniques.

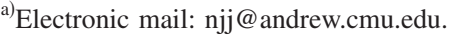

\section{EXPERIMENTAL PROCEDURE}

Polydisperse nanoparticles of composition $\left(\mathrm{Fe}_{50}\right.$ $\left.\mathrm{Co}_{50}\right)_{97} \mathrm{~V}_{2} \mathrm{Nb}_{1}$ were synthesized in a $50 \mathrm{~kW}, 3 \mathrm{MHz}$ rf Tekna induction plasma torch. These particles were then oxidized isochronally for $2 \mathrm{~h}$ at varying temperatures, ranging from RT to $900{ }^{\circ} \mathrm{C}$, in a box furnace. Timing began once the furnace returned to the desired temperature. After $2 \mathrm{~h}$, the samples were removed from the oven and allowed to cool in air. The progress of oxidation and the oxidation products were then analyzed by measuring changes in magnetization using a Lakeshore vibrating sample magnetometer and Rigaku powder x-ray diffractometer (XRD). The particles were also imaged using a JEOL 2000EX transmission electron microscope (TEM) to observe any faceting of the particles, both natively and after oxidation, and the formation of the oxide layer with increasing temperature. Mössbauer spectroscopy was employed to determine the occupation sites of the iron and cobalt in the oxide and to further understand the phases of oxide present in the nanoparticles.

\section{RESULTS AND DISCUSSION}

The magnetization of the nanoparticles was observed to decrease with increasing oxidation temperature, beginning most notably above $350-400{ }^{\circ} \mathrm{C}$. Before this, the oxidation seems only to progress to the same point of magnetization, indicating the oxide is limited by diffusion through the oxide layer at these lower temperatures. This is consistent with observations that the nanoparticles are essentially passivated by the thin oxide at RT. For low temperature applications this oxide shell means that other passivating coatings, like $\mathrm{C}$, are unnecessary. ${ }^{8}$ 


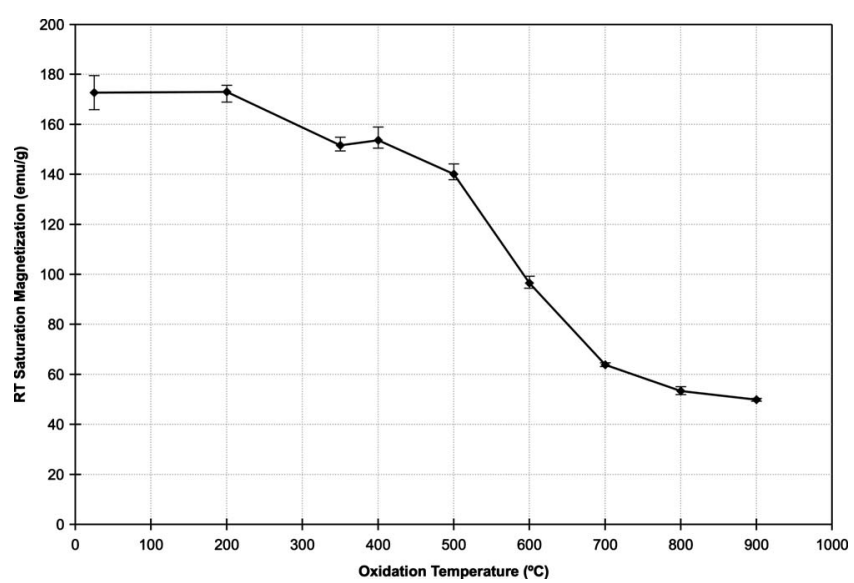

FIG. 1. RT nanoparticle saturation magnetization as a function of $2 \mathrm{~h}$ oxidation temperature.

As the oxidation temperature approached $600{ }^{\circ} \mathrm{C}$, however, an inflection point can be seen in the magnetization versus oxidation temperature data (Fig. 1). This indicates a change in the mode of oxidation, either by altering the oxide species or a change in the diffusion rate due to the building up of an oxide layer. Through XRD analysis, we observe the rapid sharpening of peaks after $400{ }^{\circ} \mathrm{C}$ along with the introduction of new oxide peaks, indicating that the oxide phase is increasing in thickness. There is also some shifting in peaks, which can either be attributed to the introduction of strains due to the growing oxide-FeCo interface or the changing of phases during oxidation. The different possible phases are magnetite, hematite, maghemite, and wustite.

In Fig. 2, TEM images show that the minimally oxidized particles are faceted along the low energy crystallographic faces, previously shown to be the (100) and (110) FeCo planes rather than (100) and (111) facets. ${ }^{1,9,10}$ The more heavily oxidized particles become more rounded (Fig. 2). At RT, the particles form a passivating oxide shell that protects the core from further oxidation; this is responsible for the slight decrease in specific magnetization from literature reported values for FeCo. The average oxide shell thickness is $3 \mathrm{~nm}$, and is relatively independent of the particle size (the oxide shell thickness ranges from about $2.5-4 \mathrm{~nm})$. FeCo

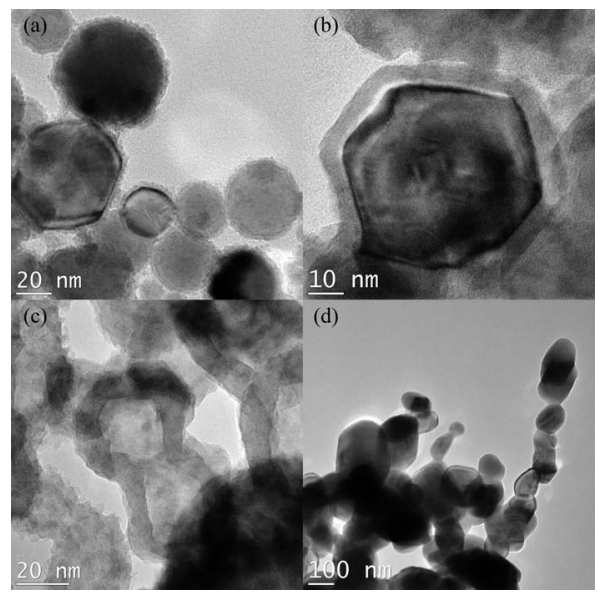

FIG. 2. TEM bright field micrographs of FeCo nanoparticles oxidized for 2 $\mathrm{h}$ at (a) RT, (b) $200{ }^{\circ} \mathrm{C}$, (c) $350{ }^{\circ} \mathrm{C}$, and (d) $900{ }^{\circ} \mathrm{C}$. cores are observed to have an average diameter of $42 \mathrm{~nm}$ but with a large spread from 25 to over $60 \mathrm{~nm}$. The massthickness contrast present inside the FeCo cores of Fig. 2(a) can be attributed to the underlying faceting of the particles.

The nanoparticles oxidized at $200{ }^{\circ} \mathrm{C}$ show a faceted FeCo core with an oxide layer that follows the core faceting. The oxide layer thickness has practically doubled and is still quite invariant with particle size $(\sim 1 \mathrm{~nm}$ variance). Some of the smallest particles are already oxidized through, and show a lighter center, indicating a voided region. Ignoring these small particles, the FeCo core has decreased to $36 \mathrm{~nm}$, which is consistent with the increase in the oxide shell thickness to $\sim 5.7 \mathrm{~nm}$.

The nanoparticles oxidized at $350{ }^{\circ} \mathrm{C}$ show a core with less contrast (like the small particles at $200{ }^{\circ} \mathrm{C}$ ), possibly indicating a voided region. The oxide has begun to round, similar to that seen in Ref. 11 for iron nanoparticles. The metal cations are therefore determined to be the mobile species during oxidation, diffusing out of the core and oxidizing at the gas-oxide interface, as seen by the coalescence of voids in the core. The oxide shell is now about $9 \mathrm{~nm}$ and the FeCo core has decreased to $25 \mathrm{~nm}$.

At the $900{ }^{\circ} \mathrm{C}$ oxidizing temperature, the nanoparticles appear very rounded and are sometimes oval-shaped. At this same temperature the saturation magnetization curve has begun to flatten out, indicating that oxidation has been completed. The nanoparticles are also much larger than in the other micrographs, about $127 \mathrm{~nm}$ in diameter, indicating that sintering of particles has taken place, and the voids have therefore been removed. There is no apparent FeCo core remaining in these nanoparticles.

Looking more closely at the magnetization curve in Fig. 1, the RT nanoparticles have a specific magnetization lower than what is predicted for an $\mathrm{Fe}_{50} \mathrm{Co}_{50}$ alloy. Using literature values for the specific magnetization of FeCo and magnetite, at RT there is about $42 \%$ magnetite in the nanoparticles, which initially seems inconsistent with the values measured from TEM. The magnetization curve, however, was averaged over a greater particle distribution, and may therefore have many more small particles which are more fully oxidized than the representative TEM images. At $900{ }^{\circ} \mathrm{C}$, the specific magnetization of the nanoparticles is below that of magnetite, indicating that other oxides have formed in the particles, as observed by XRD, and are decreasing the overall moment of the sample.

As observed through Mössbauer spectroscopy (see Fig. 3 ), the RT nanoparticles showed a single sextet with a magnetic hyperfine field (BHF) consistent with that of $\mathrm{FeCo}$ $(\mathrm{BHF}=35.5 \mathrm{~T})$. After being oxidized at $350{ }^{\circ} \mathrm{C}$ for two hours, though, the core BHF decreased to $33.1 \mathrm{~T}$, which can be attributed to an increase in Co composition, ${ }^{12}$ and three sextets were seen: one sextet for Co-rich FeCo and two sextets for the octahedral and tetrahedral sites of magnetite. While the decrease in the core BHF could also be attributed to an increase in $\mathrm{Fe}$, rather than an increase in Co, unpublished thin film compositional studies have shown Fe to be the mobile species, thus indicating that the decrease in BHF is due to an enrichment of Co in the core. Given these sextets and their specific BHF's, it seems reasonable to conclude 

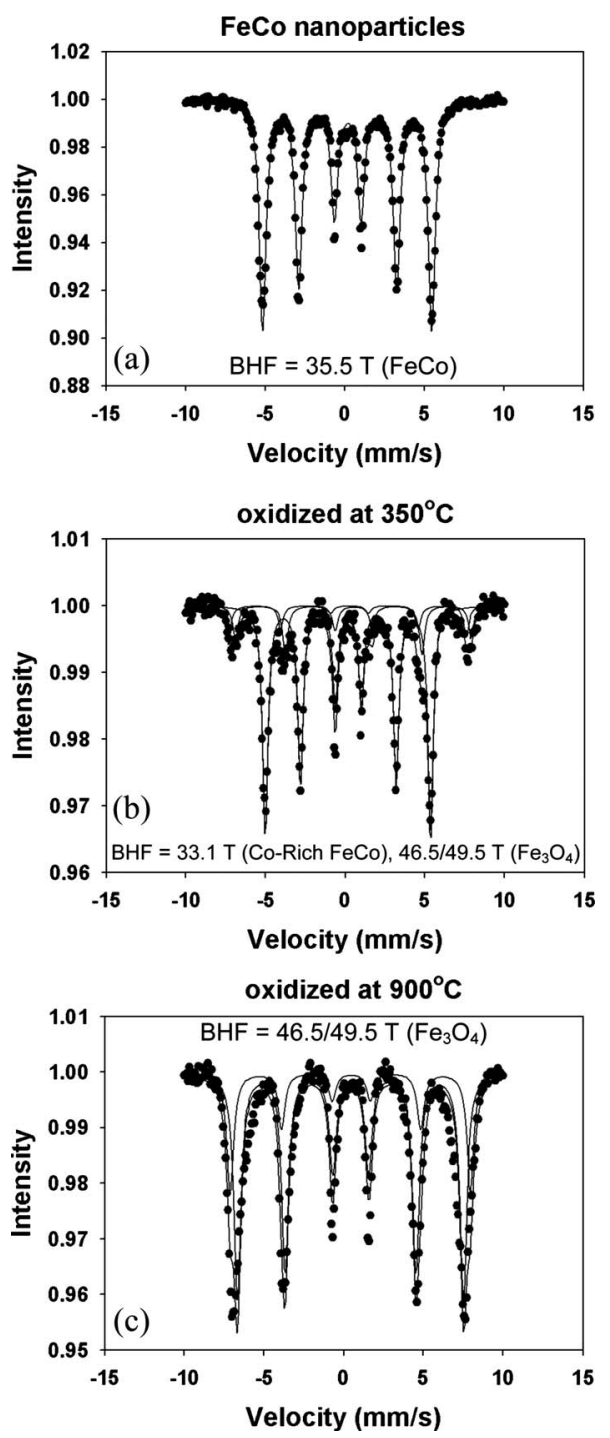

FIG. 3. Mössbauer spectrums of FeCo nanoparticles oxidized for $2 \mathrm{~h}$ at (a) RT, (b) $350{ }^{\circ} \mathrm{C}$, and (c) $900{ }^{\circ} \mathrm{C}$

that iron is the mobile species being oxidized, leaving the core richer in cobalt as oxidation progresses. The magnetite $\mathrm{BHF}$ values calculated here $(\mathrm{BHF}=46.5 / 49.5 \mathrm{~T})$ are different from those seen for cobalt-ferrite $(\mathrm{BHF}=47.5 / 50.5 \mathrm{~T})$, so cobalt does not enter the structure of magnetite. ${ }^{13}$ After oxidation at $900{ }^{\circ} \mathrm{C}$, only two sextets were seen, corresponding to the two occupation sites of magnetite, showing that all the iron remaining in the core at $350^{\circ} \mathrm{C}$ has been oxidized to form magnetite.

\section{CONCLUSIONS}

FeCo nanoparticles oxidize minimally until around $350-400{ }^{\circ} \mathrm{C}$, at which point the diffusivity of the mobile iron cations are able to penetrate through the growing oxide layer. This initial oxide layer is approximately $3 \mathrm{~nm}$ thick, with an FeCo core of $42 \mathrm{~nm}$. At this point the specific magnetization of the powders begins to decrease due to the introduction of magnetic oxides with a lower net moment than $\mathrm{FeCo}$, the oxide layer growing to $9 \mathrm{~nm}$ thick by $350{ }^{\circ} \mathrm{C}$ and the core decreasing to a diameter of $25 \mathrm{~nm}$. The oxidation tails-off above $900{ }^{\circ} \mathrm{C}$, where the particles are all oxidized and begin to sinter, increasing the particle size threefold. The growing oxide is mainly magnetite, with cobalt oxidizing separately.

\section{ACKNOWLEDGMENTS}

N.J.J., K.L.M., D.E.L., and M.E.M. would like to acknowledge the support of NSF under Grant No. DMR 0804020 and the Data Storage Systems Center. N.J.J. gratefully acknowledges support from a DOD SMART scholarship.

${ }^{1}$ K. N. Collier, N. J. Jones, K. J. Miller, Y. L. Qin, D. E. Laughlin, and M. E. McHenry, J. Appl. Phys. 105, 07A328 (2009).

${ }^{2}$ K. J. Miller, K. N. Collier, H. B. Soll-Morris, R. Swaminathan, and M. E. McHenry, J. Appl. Phys. 105, 07E714 (2009).

${ }^{3}$ B. D. Cullity and C. D. Graham, Introduction to Magnetic Materials, 2nd ed. (Wiley, New York, 2009).

${ }^{4}$ S. Chikazumi, Physics of Ferromagnetism (Oxford University Press, New York, 2002).

${ }^{5}$ J. McCord and J. Paul, IEEE Trans. Magn. 39, 2359 (2003).

${ }^{6}$ Z. Turgut, N. T. Nuhfer, H. R. Piehler, and M. E. McHenry, J. Appl. Phys. 85, 4406 (1999).

${ }^{7}$ Z. Turgut, J. C. Horwath, M. Huang, and J. E. Coate, J. Appl. Phys. 105, 07A330 (2009).

${ }^{8}$ Z. Turgut, M.-Q. Huang, K. Gallagher, S. A. Majetich, and M. E. McHenry, J. Appl. Phys. 81, 4039 (1997).

${ }^{9}$ R. Swaminathan, M. A. Willard, and M. E. McHenry, Acta Mater. 54, 807 (2006).

${ }^{10}$ R. Swaminathan, M. E. McHenry, S. Calvin, M. Sorescu, and L. Diamandescu, Proceedings of the ninth International Conference on Ferrites, American Ceramic Society, 2005 (unpublished), pp. 847-852.

${ }^{11}$ C. M. Wang, D. R. Baer, J. E. Amonette, M. H. Engelhard, Y. Qiang, and J. Antony, Nanotechnology 18, 255603 (2007).

${ }^{12}$ H. H. Hamdeh, B. Fultz, and D. H. Pearson, Phys. Rev. B 39, 11233 (1989).

${ }^{13}$ M. Sorescu, A. Grabias, D. Tarabasanu-Mihaila, and L. Diamandescu, J. Mater. Synth. Process. 9, 119 (2001). 